\title{
Neuroinflammation links COVID-19 and fragile $X$ syndrome: Role of MMP-9, IGF-1, IL-10, metformin, statins and curcumin
}

\author{
Marcos Altable
}

Funding: The author(s) received no specific funding for this work.

Potential competing interests: The author(s) declared that no potential competing interests exist.

\section{Abstract}

There is no existing specific treatment for COVID-19, so it is imperative to understand the pathophysiological pathways for the development of successful treatment. SARS-CoV-2 infection leads to the release of inflammatory cytokines which can result in an excessive inflammatory reaction or cytokine storm leading to worsening prognosis. These processes are mediated by several signalling pathways such us PI3K/Akt/mTOR and Ras/MAPK/ERK cascades, GSK3 kinase, and NF-kB. In fragile X syndrome (FXS), the most common cause of inherited mental retardation and the most common genetic form of autism spectrum disorder, those signalling pathways as well as IGF-1 are upregulated, leading to increase of metalloproteinase 9 (MMP-9) levels, tissue damage, pro-inflammatory state and blood-brain barrier disruption. The number of links found between FXS and COVID-19 suggests they share pathophysiological mechanisms. FXS would be an important risk factor for developing a severe form of COVID-19 and in turn, SARS-CoV2 could contribute to worsening the symptoms and prognosis of patients with FXS as well as asymptomatic Fmr1 premutation carriers. These asymptomatic individuals could explain a proportion of the COVID-19 cases that, without other underlying pathologies, develop cytokine storm and severe disease. With all the above, deep study of these processes that involve both FXS and COVID-19, would facilitate the discovery of potential therapeutic targets and drug development for these diseases. The potential mechanisms of metformin, trofinetide, statins, curcumin and IL-10 affecting different points of these ways are discussed further here.

\section{Introduction}

COVID-19 is an acute respiratory disease caused by a new coronavirus (SARS-CoV-2) first identified in December 2019 in Wuhan, Hubei province, China, spreading around the world to become a pandemic [1]. Globally, at February 2021, there have been 109.217.366 confirmed cases of COVID-19, including 2.413.912 deaths, reported to WHO. [2]. Since there is no existing treatment specific for COVID-19, development of successful treatment is an imperative. SARS-CoV-2 enters host cells by endocytosis using ACE-2 as receptor. Once inside begins the translation of proteins and replication of RNA [3]. The virus activates antigen-presenting cells (APC), which consequently stimulated humoral and cellular immunity systems as well as mitogen-activated protein kinase (MAPK) pathway and nuclear factor kappa B (NF-kB) signalling pathway, 
which regulate gene expression and alter immune cell differentiation [4]. This results in a release of pro-inflammatory cytokines that can lead to cytokine storm [5]. Many COVID-19 patients develop acute respiratory distress syndrome (ARDS) which leads to pulmonary edema and lung failure, and have liver, heart, and kidney damages [4], as a result of severe inflammatory reaction or hyperimmune response (cytokine storm) associated with pneumonia in critically ill patients [6].

Fragile-X syndrome (FXS), the most common cause of inherited mental retardation [7], and the most common genetic form of autism spectrum disorder [8], is caused by a lack or deficiency of the protein from Fmr1, known as fragile X mental retardation protein (FMRP). Autism spectrum disorder (ASD) occurs in approximately $60 \%$ of boys and $20 \%$ of girls with this syndrome. FXS occurs when an individual has a full mutation CGG-repeat expansion (>200 CGG repeats and methylated) in the $5^{\prime}$ non-coding portion of the Fmr1 gene at Xq27.3. There is a range of FMRP deficiency related to CGGrepeat/methylation status, with those having size and/or methylation mosaicism generally producing higher FMRP levels than those with the full mutation and fully methylated alleles [9]. Females with the full mutation generally have higher FMRP levels than males, due to the presence of a second X chromosome [7]. FXS affects one in 4,000 men and one in 8,000 women, but it may be more common when considering mild intellectual disability and conduct disorders [10]. In the general population, premutation occurs in one of 113 to 255 women and in one of 260 to 813 men [10]. FMRP is an mRNA binding protein that regulates protein translation, usually as a suppressor. It is estimated to bind $5 \%$ of mRNA in the mammalian brain [11] and is known for its role in synaptic plasticity, learning, and memory [12]. FMRP also regulates translation directly or indirectly at the level of initiation, RNA splicing, editing, nuclear export, and N6-methyladenosine modifications [13]. The loss of FMRP expression results in the cognitive and behavioral deficits associated with FXS [14]. FXS and autism share several commonalities including reduced cerebellar volume, altered dendritic spine morphology, presence of seizures and mental retardation [15]. Across species, FMRP is ubiquitously expressed but highly enriched in the brain, mirroring the fact that morphological and functional defects appear to be most prominent in, although not restricted to, the central nervous system (CNS) [16].

Other conditions have been related with FXS, such as premature ovarian failure, fragile $\mathrm{X}$ tremor ataxia syndrome (FXTAS), Parkinson's disease, cancer, immune disease, schizophrenia, bipolar disorder and major depressive disorder [17].

\section{Metalloproteinase 9 (MMP-9)}

Both Fmr1 knockout $(\mathrm{KO})$ mice and patients with FXS show altered neuronal dendritic spine morphology which is under the control of the PI3K/Akt/mTOR and Ras/MAPK signalling pathways [18]. Loss of FMRP results in hyperactivation of the mammalian/mechanistic target of rapamycin complex 1 (mTORC1) and the extracellular-signal-regulated kinase (ERK) signalling pathways [19] leading to higher production of pro-inflammatory cytokines and enhanced immune response. Consistent with increased ERK activity, eukaryotic initiation factor 4E (eIF4E) phosphorylation is elevated in the brains of individuals with FXS, thereby promoting the translation of the mRNA encoding matrix metalloproteinase 9 (MMP-9), which is also elevated in the brains of individuals with FXS [19]. Indeed, in Fmr1-null neurons, an increased level of phosphorylation of the serine/threonine kinase S6K1 has been observed. S6K1 is a common target of the mTORC1 and ERK pathways that are deregulated in the absence of FMRP [10].

It is known that SARS-CoV-2 infection leads to activation of alveolar macrophages and lung epithelial cells releasing pro- 
inflammatory cytokines that lead to enhanced endothelial permeability, allowing viral dissemination and infiltration of neutrophils and inflammatory monocytes. These cytokines stimulate the bone marrow to produce and release immature granulocytes, that return to the lung and further increase inflammation, leading to cytokine storm and ARDS [20]. Proinflammatory cytokines induce metalloproteinases (MMPs) overexpression and increase its activity. Similarly, MMPs is secreted at sites of lung inflammation in the extracellular matrix leading to release bioactive chemokines with inflammatory properties [21]. Thus, MMPs contribute to the inflammatory positive feedback loop (cytokine storm). In acute lung injury, metalloproteinase 9 (MMP-9) released from neutrophils promotes inflammation and degradation of the alveolar capillary barrier, further stimulating migration of inflammatory cells and destruction of lung tissue [22]. In addition, MMP-9 secretion is increased in coronavirus infection of human monocytes [23]. Hsu et al. reported a marked increase in plasma MMP-9 activity in critically ill patients who developed ARDS, strongly positively correlated with severity [24]. The same relationship has been described in SARS-CoV-2 [25].

At the neurological level, it is though SARS-CoV-2 can infect the central nervous system (CNS). Neurological symptoms observed in patients with COVID-19 [26,27], suggest that the involvement of the CNS could be similar to that generated in other organs of the body, which could be attributed to invasion by hematogenous route, whose most probable mechanisms could be severe systemic inflammation and disruption of the blood-brain barrier (BBB) since endothelium and smooth muscle express ACE-2. On the other hand, it is known that human coronaviruses can penetrate the CNS through the olfactory nerve and olfactory bulb neuroepithelium [28]. Then, SARS-CoV-2 could spread via retrograde neuronal mechanism where it invades the peripheral neurons and ultimately invades the CNS by spreading through the connected network of neuronal transport machinery [29]. At the CNS, pro-inflammatory cytokines, such as MMP-9, could induce neuroinflammation and neuronal damage. MMP-9 degrades components of the extracellular matrix, including proteins that are important for synaptic function and maturation, which have been implicated in FXS and ASD. In the brain, MMP-9 controls synaptic plasticity, and thus learning and memory formation [10]. Indeed, knockout of MMP-9 rescues the majority of known cellular and behavioural phenotypes in $\mathrm{Fmr1}^{-/ y}$ mice [19]. As mentioned, MMP-9 is released by neutrophils and macrophages during COVID-19 and strongly related to the severity and presence of respiratory failure, showing an early increase in COVID-19 [25]. Besides, macrophage-derived MMP-9 has been reported to be essential for T-cell infiltration into the vessel wall [30].

Furthermore, endothelial cells, located in the inner surface of vessels, secrete MMP-9 under inflammatory conditions [31]. Similarly, in pathological conditions, such as viral infections [32] and cerebrovascular diseases, mitogen-activated protein kinase kinase kinase kinase 4 (MAP4K4) leads to MMP-9 increase [33]. Previous reports have suggested that endothelial MAP4K4, expressed broadly in the brain, enhances the upregulation of leukocyte adhesion molecule, promotes monocyte infiltration, and increases chemokine leading to a rapid vascular inflammation expression $[34,35]$. Zou et al. have described that MAP4K4 triggers inflammatory vascular damage, neurological impairment, brain edema and blood-brain barrier damage. It also upregulates the expression of phosphorylated NF-kB and MMP-9, and degrades tight junction proteins (ZO-1 and claudin 5) of epithelia and BBB [33]. Bleau et al. [36] showed that coronavirus can increase BBB permeability in impairing tight endothelial junction ZO-1, VE-cadherin, and occludin expression in the brain. Loss of tight junctions in several viral infections can indirectly result from virus-induced barrier-dysregulating cytokines or chemokines, such as IL-6, TNF- $\alpha$, CCL2, or CXCL10 (Th1 type response) by brain microvascular cells (BMECs) themselves or other 
CNS cell types [36]. That is, a Th1 type response. In fact, Bleu et al. also demonstrated that IFN- $\beta$ production of endothelial cells (Th2 type response) is impaired by coronavirus.

\section{Insulin-like growth factor 1 (IGF-1)}

In brain, insulin-like growth factor 1 (IGF-1) plays a role in growth and development of all major CNS cell types and their synapse maturation. Increase in the IGF-1 pathway is associated to neuronal developmental impairment and, in particular, to autism spectrum disorder (ASD) and FXS [10]. IGF-1 exerts its function by interacting with its receptor IGF receptor 1 (IGF1R). In the mouse model of FXS, decreasing the levels of IGF1R corrects a number of phenotypic features [18]. Increased IGF-1 enhances PI3K/Akt/mTOR and Ras/MAPK signalling pathways leading to release of pro-inflammatory cytokines (IL-1ß, IFN- $\gamma$ ) and fibrinogen, that induce endothelial disfunction and hyperinflammatory state (cytokine storm). Trofinetide is a neurotrophic peptide derived from IGF-1 that shows a long half-life and is well tolerated. It acts regulating neuroinflammation, preventing apoptosis, and being neuroprotective. It normalizes brain IGF-1 (potentially a key molecular pathology), abrogates abnormalities in PI3K/Akt/mTOR and Ras/MAPK signalling implicated in neuronal plasticity, the dendritic spine morphology that accompanies abnormal plasticity, and rescues cognition, social behavior, hyperactivity, and species-typical activities. Furthermore, it is postulated to diminish neuroinflammation, reduce microglial activation and astrogliosis, and restore the enhanced excitatory/inhibitory (glutamate/GABA) neuronal signalling observed in FXS [37]. Besides, in inflammation, stimulation of the IGF-1R activates the PI3K/AKT signalling pathway, inducing AKT activation and the downstream IL-17-mediated inflammatory pathway [38].

Patients with acute respiratory distress syndrome (ARDS) have been shown to have increased IGF-1 and IGF-1R staining in lung tissue specimens. Rising levels of IGF-1 in bronchioalveolar fluid (BAL) and increased IGF-1 mRNA expression in lung tissues have been found in ARDS [38]. Similarly, Piñeiro-Hermida et al. demonstrated that IGF1-R deficiency, was associated with improved survival, reduced vascular fragility and permeability, and less inflammatory cell lung infiltrate in lung injury [39]. In H1N1 influenza infection (Spanish flu), it has been seen that increase of IGF-1 enhances PI3K/AKT and MAPK signalling, lung injury and mortality [40].

\section{Metformin}

MMP-9 inhibitors, such as aprotinin, have been proposed as a treatment in COVID-19 [21]. Administration of metformin, an FDA-approved anti-diabetic drug, in FXS (Fmr1 knockout flies) [41], leads to an amelioration of memory defects [42]. Metformin normalizes mTOR and ERK signalling, eIF4E phosphorylation and the expression of MMP-9, and has been shown to improve FXS symptoms [19]. Metformin use is associated with reduced mortality rate from coronavirus disease 2019 (COVID-19) infection [43]. Several mechanisms have been proposed to explain this effect: inhibition of mTOR pathway and prevention of immune hyperactivation; action on endosomal $\mathrm{Na}^{+} / \mathrm{H}^{+}$exchanger, thereby increasing cellular $\mathrm{pH}$ and interference with viral endocytic cycle; anti-inflammatory properties; reduction in neutrophils; inhibition of mitochondrial complex 1 , suppression of mitochondrial reactive oxygen species (ROS) signalling, necessary for the opening of $\mathrm{Ca}^{2+}$ release-activated $\mathrm{Ca}^{2+}$ (CRAC) channels in the generation of IL-6 from alveolar macrophages; among others [43]. Interestedly, markers of oxidative stress are associated with various neurological diseases, ageing, and cases of FXS with ASD [44]. Thus, individuals with FXS exhibit higher levels of oxidative stress. Indeed, there is a close relationship between reactive oxygen species (ROS) and FMRP deficiencies [45].

On the other hand, metformin is known to inhibit IGF-1 by the reversal of hyperinsulinemia. It ameliorates the pulmonary 
injury in an experimental model of ARS by PI3K signalling inhibition and IGF-1 decrease [46].

\section{Statins}

It has been described that treatment with the cholesterol-lowering agent lovastatin improve behavioural outcome in FXS patients [47]. Concerning COVID-19, statins are capable of inducing shifts from Th1 cytokine production to Th2 type cytokine secretion, (IL-4, IL-5, IL-9, IL-10, and IFN- $\alpha / \beta$ instead of IL- 6 IL-1B, IL-8, and IFN- $\gamma$ ), ameliorating cytokine storm and macrophage activation, and switching immune response in anti-inflammatory and pro-repair activity [48]. Therefore, statins not only block virus replication upon antiviral activity but also reduces the harmful effects of inflammation on the host [49]. Moreover, they reduce the synthesis of cholesterol, that is the main substrate for aldosterone synthesis in the Ang-Il function. Statins also inhibit AKT/mTOR, NF-kB and Ras-MAPK-ERK pathways [50], avoiding inflammation, endothelial dysfunction and increased vascular permeability that can lead to multi-organ failure; protein overexpression by increasing translation and transcription; and elevation of intracellular calcium. These events might improve FXS symptoms and decrease SARS-CoV-2 infectivity and COVID-19 severity.

\section{Curcumin}

Curcumin or curcumol (Cur), isolated from the traditional Chinese medical plant Rhizoma Curcumae, is broadly studied in oncology due to its anti-tumoral activity [51]. Curcumin have been reported for neuroprotective effect in several neurological disorder also such as Alzheimer's, Parkinson's, frontotemporal dementia, and Huntington's disease. Administration of curcumin improved the cell viability through various mechanism including inhibition of amyloid formation and its toxicity in Alzheimer disease, reduction of oxidative damage, inflammation, and synuclein protein aggregation in Parkinson disease, and decrease of aggregation of tau proteins in frontotemporal dementia. It also facilitates anti-anxiety activity by decrease level of TNF- $\alpha$ and MMP-9 in ASD [52]. Recently, it has been described that curcumin ameliorates defects in FXS by binding CGG repeats RNA, which are the etiology of FSX and related diseases such as ASD and fragile X-associated tremor ataxia syndrome (FXTAS). Besides, curcumin inhibits PI3K/AKT and ERK/NF-kB signalling pathways [53]. Curcumin also downregulates glycogen synthase kinase 3 (GSK-3) and $\beta$-catenin pathway [54], that are involved in SARS-CoV-19 infectivity [55], COVID-19 boosted inflammation [56], BBB disruption [57] and FXS pathophysiology [58]. Interestingly, chloroquine inhibits GSK-3 in lung epithelial and immune cells, so GSK3 has been considered as a central axis in inflammation during SARS-CoV-2 infection [55]. Regarding GSK-3 features, FMRP is responsible for inhibiting mRNA transcription of GSK-3 [58]. Therefore, in FXS, the absence of FMRP inhibition leads to upregulation of GSK-3. In fact, GSK-3 inhibition corrects pathophysiology in a mouse model of fragile X syndrome [59]. It is involved in pathways that promote inflammation and induces IL-6 [58] and IFN- $\gamma$ production [56]. The SARs CoV-1 N protein is phosphorylated by GSK-3, an event that is needed for viral replication. Other studies have shown that GSK-3 negatively regulates T-cell proliferation and function [56]. Indeed, it has been shown that GSK-3 is needed by SARS-CoV replication and its inhibition had been proposed as a COVID-19 treatment [56].

Thus, GSK-3 pathway might be involved in pathophysiology of both COVID-19 and FXS, and they would act reciprocally. GSK-3 would improve SARS-CoV-2 infectivity and worse FXS symptoms. Summary, curcumin decreases TNF- $\alpha$, MMP-9 and PI3K/AKT and ERK/NF-KB signalling. Considering all above mentioned, it improves inflammation, tissue damage and deleterious effects of IFG-1, becoming a possible therapeutic agent in both COVID-19 and FXS. 


\section{Interleukink-10 (IL-10)}

It is also noticeable that administration of IL-10 en mice model of FXS is able to reverse the learning and memory impairment, as well as in mice with GSK-3 overexpression. These results again indicate that inflammation is involved in the FXS phenotype [60]. On the other hand, administration of IL-10 could exert therapeutic effects on COVID-19, due to its anti-inflammatory activity of IL-10 (Th2 immune response).

\section{Conclusion}

There is still no effective drug for treating both COVID-19 and FXS. Identify critical molecular targets and the role they play in infection is needed for drug development. FXS and COVID-19 share links in their pathophysiology that include neuroinflammation, and inhibition at these levels could become therapeutic targets. Thus, known drugs as metformin, trofinetide, statins, curcumin and IL-10, might implement the COVID-19 and FXS treatment.

Indeed, we posit FXS would be an important risk factor for developing a severe form of COVID-19 and in turn, SARS-CoV2 could contribute to worsening the symptoms and prognosis of patients with FXS and asymptomatic Fmr1 premutation carriers.

This fact leads us to suggest that a proportion of unexplained severe COVID-19 cases in young people could correspond to asymptomatic premutation carriers, what we could know as 'fragile storm'.

\section{Conflict of interest statement}

Marcos Altable and Juan Moisés de la Serna have no conflicts of interest to disclose regarding the manuscript. The authors declare that the manuscript was conducted in the absence of any commercial or financial relationships that could be construed as a potential conflict of interest. This research did not receive any specific grant from funding agencies in the public, commercial, or not-for-profit sectors

\section{References}

[1] Wu F, Zhao S, Yu B, Chen YM, Wang W, Song ZG, et al. A new coronavirus associated with human respiratory disease in China. Nature 2020;579:265-9. https://doi.org/10.1038/s41586-020-2008-3.

[2] Organization WH. WHO Coronavirus Disease (COVID-19) Dashboard. WHO Heal Emerg Dashboard 2020. https://covid19.who.int/ (accessed 7 September 2020).

[3] Cava C, Bertoli G, Castiglioni I. A protein interaction map identifies existing drugs targeting SARS-CoV-2. BMC Pharmacol Toxicol 2020;21:65. https://doi.org/10.1186/s40360-020-00444-z.

[4] Wu D, Yang XO. TH17 responses in cytokine storm of COVID-19: An emerging target of JAK2 inhibitor Fedratinib. J Microbiol Immunol Infect 2020;53:368-70. https://doi.org/10.1016/j.jmii.2020.03.005.

[5] Berretta AA, Duarte Silveira MA, Cóndor Capcha JM, De Jong D. Propolis and its potential against SARS-CoV-2 infection mechanisms and COVID-19 disease. Biomed Pharmacother 2020;131:110622.

https://doi.org/10.1016/j.biopha.2020.110622.

[6] Sahebnasagh A, Saghafi F, Avan R, Khoshi A, Khataminia M, Safdari M, et al. The prophylaxis and treatment potential of supplements for COVID-19. Eur J Pharmacol 2020:173530. https://doi.org/10.1016/j.ejphar.2020.173530. [7] Tassone F, Hagerman RJ, Iklé DN, Dyer PN, Lampe M, Willemsen R, et al. FMRP expression as a potential prognostic indicator in fragile X syndrome. Am J Med Genet 1999;84:250-61.

[8] Jacquemont S, Hagerman RJ, Hagerman PJ, Leehey MA. Fragile-X syndrome and fragile X-associated 
tremor/ataxia syndrome: two faces of FMR1. Lancet Neurol 2007;6:45-55. https://doi.org/10.1016/S1474-4422(06)70676-7. [9] Hagerman RJ, Hull CE, Safanda JF, Carpenter I, Staley LW, O'Connor RA, et al. High functioning fragile X males: Demonstration of an unmethylated fully expanded FMR-1 mutation associated with protein expression. Am J Med Genet 1994;51:298-308. https://doi.org/10.1002/ajmg.1320510404.

[10] Castagnola S, Bardoni B, Maurin T. The search for an effective therapy to treat fragile $X$ syndrome: dream or reality? Front Synaptic Neurosci 2017;9:15.

[11] Darnell JC, Klann E. The translation of translational control by FMRP: therapeutic targets for FXS. Nat Neurosci 2013;16:1530-6.

[12] Deng PY, Sojka D, Klyachko VA. Abnormal presynaptic short-term plasticity and information processing in a mouse model of fragile X syndrome. J Neurosci 2011;31:10971-82. https://doi.org/10.1523/JNEUROSCI.2021-11.2011.

[13] Shu HR, Donnard E, Liu B, Wang R, Richter J. FMRP Links Optimal Codons to mRNA stability in Neurons. BioRxiv 2020:801449.

[14] Oostra BA, Willemsen R. FMR1: A gene with three faces. Biochim Biophys Acta - Gen Subj 2009;1790:467-77. https://doi.org/10.1016/j.bbagen.2009.02.007.

[15] Fatemi SH, Folsom TD, Liesch SB, Kneeland RE, Karkhane Yousefi M, Thuras PD. The effects of prenatal H1N1 infection at E16 on FMRP, glutamate, GABA, and reelin signaling systems in developing murine cerebellum. J Neurosci Res 2017;95:1110-22. https://doi.org/10.1002/jnr.23949.

[16] Logan MA. Fragile phagocytes: FMRP positively regulates engulfment activity. J Cell Biol 2017;216:531-3. https://doi.org/10.1083/jcb.201702034.

[17] Pasciuto E, Bagni C. SnapShot: FMRP mRNA Targets and Diseases. Cell 2014;158:1446.e1-1446.e1. https://doi.org/10.1016/j.cell.2014.08.035.

[18] Deacon RMJ, Glass L, Snape M, Hurley MJ, Altimiras FJ, Biekofsky RR, et al. NNZ-2566, a Novel Analog of (1-3) IGF-1, as a Potential Therapeutic Agent for Fragile X Syndrome. NeuroMolecular Med 2015;17:71-82. https://doi.org/10.1007/s12017-015-8341-2.

[19] Gantois I, Khoutorsky A, Popic J, Aguilar-Valles A, Freemantle E, Cao R, et al. Metformin ameliorates core deficits in a mouse model of fragile X syndrome. Nat Med 2017;23:674-7. https://doi.org/10.1038/nm.4335.

[20] Polidoro RB, Hagan RS, de Santis Santiago R, Schmidt NW. Overview: Systemic Inflammatory Response Derived From Lung Injury Caused by SARS-CoV-2 Infection Explains Severe Outcomes in COVID-19. Front Immunol 2020;11. https://doi.org/10.3389/fimmu.2020.01626.

[21] Solun B, Shoenfeld Y. Inhibition of metalloproteinases in therapy for severe lung injury due to COVID-19. Med Drug Discov 2020;7:100052. https://doi.org/10.1016/j.medidd.2020.100052.

[22] Davey A, McAuley DF, O'Kane CM. Matrix metalloproteinases in acute lung injury: Mediators of injury and drivers of repair. Eur Respir J 2011;38:959-70. https://doi.org/10.1183/09031936.00032111.

[23] Desforges M, Miletti TC, Gagnon M, Talbot PJ. Activation of human monocytes after infection by human coronavirus 229E. Virus Res 2007;130:228-40. https://doi.org/10.1016/j.virusres.2007.06.016.

[24] Hsu AT, Barrett CD, DeBusk GM, Ellson CD, Gautam S, Talmor DS, et al. Kinetics and role of plasma matrix metalloproteinase-9 expression in acute lung injury and the acute respiratory distress syndrome. Shock 2015;44:128-36. 
https://doi.org/10.1097/SHK.0000000000000386.

[25] Ueland T, Holter JC, Holten AR, Müller KE, Lind A, Bekken GK, et al. Distinct and early increase in circulating MMP9 in COVID-19 patients with respiratory failure: MMP-9 and respiratory failure in COVID-19. J Infect 2020;81:e41-3. https://doi.org/10.1016/j.jinf.2020.06.061.

[26] Mao L, Jin H, Wang M, Hu Y, Chen S, He Q, et al. Neurologic Manifestations of Hospitalized Patients with Coronavirus Disease 2019 in Wuhan, China. JAMA Neurol 2020;77:683-90.

https://doi.org/10.1001/jamaneurol.2020.1127.

[27] Moriguchi T, Harii N, Goto J, Harada D, Sugawara H, Takamino J, et al. A first case of meningitis/encephalitis associated with SARS-Coronavirus-2. Int J Infect Dis 2020;94:55-8. https://doi.org/10.1016/j.jijid.2020.03.062.

[28] Desforges M, Le Coupanec A, Stodola JK, Meessen-Pinard M, Talbot PJ. Human coronaviruses: Viral and cellular factors involved in neuroinvasiveness and neuropathogenesis. Virus Res 2014;194:145-58. https://doi.org/10.1016/j.virusres.2014.09.011.

[29] Zhou Z, Kang H, Li S, Zhao X. Understanding the neurotropic characteristics of SARS-CoV-2: from neurological manifestations of COVID-19 to potential neurotropic mechanisms. J Neurol 2020;267:2179-84. https://doi.org/10.1007/s00415-020-09929-7.

[30] Watanabe R, Maeda T, Zhang H, Berry GJ, Zeisbrich M, Brockett R, et al. MMP (matrix metalloprotease)-9-producing monocytes enable T cells to invade the vessel wall and cause vasculitis. Circ Res 2018;123:700-15.

https://doi.org/10.1161/CIRCRESAHA.118.313206.

[31] Yang YI, Estrada EY, Thompson JF, Liu W, Rosenberg GA. Matrix metalloproteinase-mediated disruption of tight junction proteins in cerebral vessels is reversed by synthetic matrix metalloproteinase inhibitor in focal ischemia in rat. $\mathrm{J}$ Cereb Blood Flow \\& Metab 2007;27:697-709.

[32] Strang BL, Asquith CRM, Moshrif HF, Ho CMK, Zuercher WJ, Al-Ali H. Identification of lead anti-human cytomegalovirus compounds targeting map4k4 via machine learning analysis of kinase inhibitor screening data. PLoS One 2018;13. https://doi.org/10.1371/journal.pone.0201321.

[33] Zou Z, Dong Y-S, Liu D-D, Li G, Hao G-Z, Gao X, et al. MAP4K4 induces early blood-brain barrier damage in a murine subarachnoid hemorrhage model. Neural Regen Res 2021;16:325. https://doi.org/10.4103/1673-5374.290904.

[34] Pannekoek WJ, Linnemann JR, Brouwer PM, Bos JL, Rehmann H. Rap1 and Rap2 Antagonistically Control Endothelial Barrier Resistance. PLoS One 2013;8. https://doi.org/10.1371/journal.pone.0057903.

[35] Vitorino P, Yeung S, Crow A, Bakke J, Smyczek T, West K, et al. MAP4K4 regulates integrin-FERM binding to control endothelial cell motility. Nature 2015;519:425-30. https://doi.org/10.1038/nature14323.

[36] Bleau C, Filliol A, Samson M, Lamontagne L. Brain Invasion by Mouse Hepatitis Virus Depends on Impairment of Tight Junctions and Beta Interferon Production in Brain Microvascular Endothelial Cells. J Virol. 2015;89(19):9896-9908. doi:10.1128/JVI.01501-15

[37] Berry-Kravis E, Horrigan JP, Tartaglia N, Hagerman R, Kolevzon A, Erickson CA, et al. A Double-Blind, Randomized, Placebo-Controlled Clinical Study of Trofinetide in the Treatment of Fragile X Syndrome. Pediatr Neurol 2020;110:30-41. https://doi.org/10.1016/j.pediatrneurol.2020.04.019.

[38] Winn BJ. Is there a role for insulin-like growth factor inhibition in the treatment of COVID-19-related adult respiratory 
distress syndrome? Med Hypotheses 2020;144. https://doi.org/10.1016/j.mehy.2020.110167.

[39] Piñeiro-Hermida S, López IP, Alfaro-Arnedo E, Torrens R, Iñiguez M, Alvarez-Erviti L, et al. IGF1R deficiency attenuates acute inflammatory response in a bleomycin-induced lung injury mouse model. Sci Rep 2017;7. https://doi.org/10.1038/s41598-017-04561-4.

[40] Li G, Zhou L, Zhang C, Shi Y, Dong D, Bai M, et al. Insulin-Like Growth Factor 1 Regulates Acute Inflammatory Lung Injury Mediated by Influenza Virus Infection. Front Microbiol 2019;10. https://doi.org/10.3389/fmicb.2019.02541.

[41] Viollet B, Guigas B, Sanz Garcia N, Leclerc J, Foretz M, Andreelli F. Cellular and molecular mechanisms of metformin: An overview. Clin Sci 2012;122:253-70. https://doi.org/10.1042/CS20110386.

[42] Monyak RE, Emerson D, Schoenfeld BP, Zheng X, Chambers DB, Rosenfelt C, et al. Insulin signaling misregulation underlies circadian and cognitive deficits in a Drosophila fragile X model. Mol Psychiatry 2017;22:1140-8. https://doi.org/10.1038/mp.2016.51.

[43] Scheen AJ. Metformin and COVID-19: From cellular mechanisms to reduced mortality. Diabetes $1 \&$ Metab 2020. [44] Romero-Zerbo Y, Decara J, El Bekay R, Sanchez-Salido L, Del Arco-Herrera I, De Fonseca FR, et al. Protective effects of melatonin against oxidative stress in Fmr1 knockout mice: A therapeutic research model for the fragile $\mathrm{X}$ syndrome. J Pineal Res 2009;46:224-34. https://doi.org/10.1111/j.1600-079X.2008.00653.x.

[45] El Bekay R, Romero-Zerbo Y, Decara J, Sanchez-Salido L, Del Arco-Herrera I, Rodríguez-De Fonseca F, et al. Enhanced markers of oxidative stress, altered antioxidants and NADPH-oxidase activation in brains from Fragile $X$ mental retardation 1-deficient mice, a pathological model for Fragile X syndrome. Eur J Neurosci 2007;26:3169-80. https://doi.org/10.1111/j.1460-9568.2007.05939.x.

[46] Xiao H, Huang X, Wang S, Liu Z, Dong R, Song D, et al. Metformin ameliorates bleomycin-induced pulmonary fibrosis in mice by suppressing IGF-1. Am J Transl Res 2020;12:940.

[47] Çaku A, Pellerin D, Bouvier P, Riou E, Corbin F. Effect of lovastatin on behavior in children and adults with fragile $X$ syndrome: An open-label study. Am J Med Genet Part A 2014;164:2834-42. https://doi.org/10.1002/ajmg.a.36750.

[48] Youssef S, Stüve O, Patarroyo JO, Ruiz PJ, Radosevich JL, Mi Hur E, et al. The HMG-CoA reductase inhibitor, atorvastatin, promotes a Th2 bias and reverses paralysis in central nervous system autoimmune disease. Nature 2002;420:78-84. https://doi.org/10.1038/nature01158.

[49] Fedson DS. A practical treatment for patients with Ebola virus disease. J Infect Dis 2015;211:661-2.

[50] Shang L, Jia SS, Jiang HM, Wang H, Xu WH, Lv CJ. Simvastatin downregulates expression of TGF- $\beta R I I$ and inhibits proliferation of A549 cells via ERK. Tumor Biol 2015;36:4819-24. https://doi.org/10.1007/s13277-015-3134-7.

[51] Li S, Zhou G, Liu W, Ye J, Yuan F, Zhang Z. Curcumol inhibits lung adenocarcinoma growth and metastasis via inactivation of PI3K/AKT and Wnt/B-catenin pathway. Oncol Res Featur Preclin Clin Cancer Ther 2020. https://doi.org/10.3727/096504020x15917007265498.

[52] Ning N, Liu S, Liu X, Tian Z, Jiang Y, Yu N, et al. Curcumol inhibits the proliferation and metastasis of melanoma via the miR-152-3p/PI3K/AKT and ERK/NF-KB signaling pathways. J Cancer 2020;11:1679-92. https://doi.org/10.7150/jca.38624.

[53] Verma AK, Khan E, Mishra SK, Mishra A, Charlet-Berguerand N, Kumar A. Curcumin Regulates the r(CGG)exp RNA Hairpin Structure and Ameliorate Defects in Fragile X-Associated Tremor Ataxia Syndrome. Front Neurosci 2020;14. 
https://doi.org/10.3389/fnins.2020.00295.

[54] Cui S, Liu Z, Tao B, Fan S, Pu Y, Meng X, et al. miR-145 attenuates cardiac fibrosis through the AKT/GSK-3 $/ \beta$ catenin signaling pathway by directly targeting SOX9 in fibroblasts. J Cell Biochem 2020:jcb.29843.

https://doi.org/10.1002/jcb.29843.

[55] Embi MN, Ganesan N, Sidek HM. Is GSK3 $\beta$ a molecular target of chloroquine treatment against COVID-19? Drug Discov Ther 2020;14:107-8. https://doi.org/10.5582/ddt.2020.03010.

[56] Rudd CE. GSK-3 Inhibition as a Therapeutic Approach Against SARs CoV2: Dual Benefit of Inhibiting Viral Replication While Potentiating the Immune Response. Front Immunol 2020;11. https://doi.org/10.3389/fimmu.2020.01638. [57] Hou Y, Hu Z, Gong X, Yang B. HSPB8 overexpression prevents disruption of blood-brain barrier after intracerebral hemorrhage in rats through Akt/GSK3\&amp;\#x3B2;/\&amp;\#x3B2;-catenin signaling pathway. Aging (Albany NY) 2020;12. https://doi.org/10.18632/aging.103773.

[58] Portis S, Giunta B, Obregon D, Tan J. The role of glycogen synthase kinase-3 signaling in neurodevelopment and fragile X syndrome. Int J Physiol Pathophysiol Pharmacol 2012;4:140.

[59] McCamphill PK, Stoppel LJ, Senter RK, Lewis MC, Heynen AJ, Stoppel DC, et al. Selective inhibition of glycogen synthase kinase $3 \alpha$ corrects pathophysiology in a mouse model of fragile X syndrome. Sci Transl Med 2020;12. https://doi.org/10.1126/scitransImed.aam8572.

[60] Worthen RJ, Garzon Zighelboim SS, Torres Jaramillo CS, Beurel E. Anti-inflammatory IL-10 administration rescues depression-associated learning and memory deficits in mice. J Neuroinflammation 2020;17.

https://doi.org/10.1186/s12974-020-01922-1. 